\title{
Controle da malária transfusional em região endêmica e não endêmica do Brasil
}

\author{
Transfusion malaria control in both endemic and non endemic \\ region of Brazil
}

\author{
Amadeo Sáez-Alquézar, Ana Mario S. do Val Romos, Silvin M. Di Santi, Maria \\ Stela Branquinho, Karin Kirchgatter, Ingrid A.C. Cordeiro, Marcin Murta, João \\ C.P. Soraiva, Solma Gomes de Oliveira, Mario Gobriella Gioelli Bochetti, \\ José Antonio Pirolla, Dalton Guerzoni e Dalton A.F. Chamone.
}

\begin{abstract}
Resumo O presente trabalho teve como objetivo estabelecer critérios adequados à triagem de doadores de sangue de regiões com características epidemiológicas distintas, para malária. Foram estudados 3 locais com critérios de seleção diferentes: São Paulo, SP (sem transmissão vetorial), Belém (baixa transmissão ativa), Matupá, Belém, PA e Peixoto de Azevedo, MT (alta transmissão ativa). A pesquisa de plasmódios foi realizada por gota espessa, QBC Test e imunofluorescência para pesquisa de antígenos, tendo sido todas as amostras negativas. Houve grande variação na positividade para anticorpos antiplasmodiais por IFl-IgG anti P. vivax e P. falciparum entre doadores aptos nos 3 locais de estudo e entre doadores aptos e inaptos em São Paulo (aptos 1,98\%, inaptos $22,3 \%, p<0.01$ ) e Belém (aptos 17,2\%, inaptos 58,3\%, $p<0.01$ ), o que atesta a validade da triagem clínico-epidemiológica realizada. Em Matupá e Peixoto de Azevedo não houve doadores inaptos e a positividade foi de $80,6 \%$. Consideramos que em bancos de sangue a triagem deve seguir critérios clínico-epidemiológicos adequados à situação de cada região. Os métodos laboratoriais de triagem, devem ser para detecção de plasmódios (gota espessa/QBC Test@ ou detecção de antígenos parasitários.
\end{abstract}

Palavras-chaves: Malária. Banco de Sangue. Sorologia.

\begin{abstract}
The aim of the present work was to establish appropriate criteria for screening of donor blood from regions with distinct Malaria epidemiological characteristics. Three locations with different screening criteria were studied: São Paulo, SP (with no vectorial transmission), Belém, PA (with low active transmission) and Matupá and Peixoto de Azevedo, MT (with high active transmission). The Malaria parasite — Plasmodium sp — was searched for by "thick film", QBC Test( and antigen Immunofluorescence test, and was not detected in any of the samples. There was, however, a great variation in the positivity of anti-plasmodial antibodies, as determined by IIF-IgG anti-P. vivax and $-P$. falciparum, between accepted donors in the 3 studied locations and between rejected and accepted donors in São Paulo (1.98\% accepted, $22.3 \%$ rejected - $p<0.01)$ and Belém (17.2\% accepted, $58.3 \%$ rejected $-p<0.01$ ). These data endorse the use of the applied clinical and epidemiological screening. In Matupá and Peixoto de Azevedo, where there was no rejected donor, the serological positivity was $80.6 \%$. We, therefore, consider that the Malaria screening in blood banks should follow clinical and epidemiological criteria suitable to each region. The laboratorial

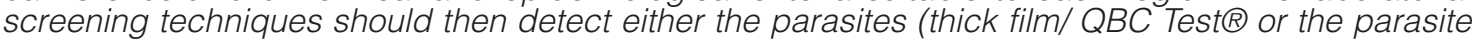
antigens.
\end{abstract}

Key-words: Malaria. Blood bank. Serology.

\footnotetext{
Fundação Pró-Sangue/Hemocentro de São Paulo (FPS/HSP), Superintendência de Controle de Endemias (SUCEN) e Instituto de Medicina Tropical da Universidade de São Paulo (IMT/USP) São Paulo, SP. Fundação Centro de Hematologia e Hemoterapia do Pará (HEMOPA) e Instituto Evandro Chagas (IEC) Belém, PA. Hospital Regional de Matupá (HRM) Matupá, MT e Hospital do Garimpeiro (HG) Peixoto de Azevedo, MT.

Apoio financeiro: Programa de Controle de Malária na Amazônia, Fundação Nacional de Saúde (PCMAM/FNS).

Endereço para correspondência: Dr. Amadeo Sáez-Alquézar. Superintendente de Sorologia e Controle de Qualidade/Fundação PróSangue Hemocentro de São Paulo. Av. Dr. Enéas de Carvalho Aguiar 155, Bloco 4, 1a andar, 05403-000 São Paulo, SP, Brasil. Tel: (011) 3061-5544. Fax: (011) 280-8317.

Recebido para publicação em 14/04/97.
} 
A transmissão da malária por via transfusional foi descrita pela primeira vez em 191127. Os dados referentes aos casos de malária transfusional são aproximados. Bruce-Chwatt, em 1985, relata pelo menos 3500 casos em um período de 65 anos 8 . A incidência média de malária transfusional nos Estados Unidos de 1972 a 1981 foi de 0,25 casos por milhão de unidades de sangue coletadas. De um total de 45 casos, $38 \%$ foram devidos a infecções por Plasmodium malariae, $29 \%$ por $P$. falciparum, $24 \%$ por $P$. vivax e $9 \%$ por $P$. ovale, com um percentual de $7 \%$ de óbitos 15 .

A transmissão de malária em transfusões ocorre normalmente através de sangue total $8 \mathrm{e}$ concentrado de hemácias 8 , podendo ocorrer com menor freqüência através de concentrado de plaquetas ${ }^{14}$, leucócitos ${ }^{12}$, crioprecipitados 26 e plasma fresco18. Os principais mecanismos envolvidos são: a infecção malárica sem sinais clínicos nos doadores e a viabilidade do Plasmodium nas amostras de sangue estocadas6.

No Brasil, embora a real incidência de malária transfusional seja desconhecida, este evento pode estar contribuindo para a disseminação da doença em áreas com transmissão ativa, onde a triagem clínico-epidemiológica não esteja sendo efetuada de maneira rigorosa. A Portaria $\mathrm{n}^{-}$ 1376 do Ministério da Saúde, de 19 de novembro de 1993, que altera a Portaria no $721 / 89$, apenas recomenda a realização de exame parasitológico/hemoscópico para malária, em regiões endêmicas com transmissão ativa e exames sorológicos em regiões endêmicas sem transmissão ativa, porém vulneráveis, propiciando condutas não uniformes em serviços de hemoterapia. Além disso, a ocorrência de baixa parasitemia em indivíduos assintomáticos 26 e o escasso conhecimento sobre a malária em áreas sem transmissão ativa, podem ocasionar a transfusão de unidades de sangue ou hemocomponentes contaminados. Aliada a estes dois fatores, a desinformação com relação à distribuição geográfica da malária, pode determinar equívocos quando da realização da triagem epidemiológica, através de questionários.

Estudo realizado em doadores da Fundação Pró-Sangue Hemocentro de São Paulo (FPS/HSP)20 em 1993, mostrou a eficácia da triagem clínico-epidemiológica para malária, através da diferença significativa observada, entre os grupos de candidatos, aptos ou inaptos, quanto à presença de anticorpos antiplasmodiais da classe IgG, utilizando-se a técnica de imunofluorescência indireta (IFI). Nesse mesmo trabalho descreve-se um caso de P. vivax detectado por QBC Test ${ }^{\circledR}$ (Quantitative Buffy Coat) em candidato a doador de plaquetas, para paciente submetido a transplante de medula óssea, ambos provenientes de Rondonópolis, MT. Este fato mostra a necessidade da padronização de procedimentos adequados, para evitar a transmissão de malária pela via transfusional, por parte de indivíduos provenientes de áreas com transmissão ativa.

O presente trabalho teve como objetivos estabelecer métodos e critérios adequados à triagem de doadores de sangue para regiões com características epidemiológicas distintas e avaliar a triagem clínico-epidemiológica dos doadores em relação à malária.

\section{MATERIAL E MÉTODOS}

Locais de estudo. Foram escolhidos três locais, diferentes entre si, tanto em relação aos aspectos epidemiológicos, quanto aos critérios utilizados na seleção dos doadores:

1. São Paulo, SP - Fundação Pró-Sangue/ Hemocentro de São Paulo (FPS/HSP). O município de São Paulo constitui área considerada sem ocorrência de transmissão vetorial de malária. No entanto, alguns fatores devem ser levados em consideração: presença de focos esporádicos e restritos a determinados locais do Estado, responsáveis por 41 casos autóctones notificados no ano de 1993 e 28 casos em 19945; ocorrência no Estado, de 27 casos notificados de malária transfusional, nos últimos doze anos5 e deslocamento de indivíduos provenientes de outras regiões do país, onde a transmissão vetorial é intensa e constante, com 552.247 casos notificados em 1994 e 555.713 casos em 19954.

2. Belém, PA - Fundação Centro de Hemoterapia e Hematologia do Pará (HEMOPA). O município de Belém possui um índice parasitário anual por mil habitantes, de 1.1, podendo ser considerado área de baixa transmissão ativa3.

3. Matupá e Peixoto de Azevedo, MT - Hospital Regional de Matupá (HRM) e Hospital do Garimpeiro (HG). Os índices parasitários anuais por mil habitantes, destes dois municípios são de 875.0 e 370.5 , respectivamente, considerados representativos de áreas com alta transmissão de malária3. 
Triagem clínico-epidemiológica. A triagem dos candidatos a doadores de sangue da FPS/HSP baseia-se nos seguintes critérios epidemiológicos: malária pregressa (exclusão definitiva); deslocamento para área de transmissão ativa sem uso de profilaxia medicamentosa (exclusão por seis meses) e com uso de profilaxia medicamentosa (exclusão por 3 anos); ex-moradores de área com transmissão ativa (exclusão por 3 anos), segundo critérios estabelecidos pelo Ministério da Saúde e American Association of Blood Banks ${ }^{11}$. Na FPS/HSP a recusa de doadores por critérios epidemiológicos relacionados à malária é de $0,62 \% 11$.

Os critérios utilizados para exclusão de doadores no HEMOPA são os determinados pelo Ministério da Saúde: febre nos últimos 30 dias, deslocamento para áreas com transmissão de malária nos últimos 6 meses e malária nos últimos 12 meses.

No HRM e HG o único critério para exclusão de doadores é febre nos últimos 30 dias.

Os dados clínico-epidemiológicos de cada doador foram obtidos através da aplicação de um questionário comum às três regiões estudadas.

Amostras. Foram ensaiadas 1.200 amostras de sangue de doadores da FPS/HSP (1164 aptos e 36 inaptos), 250 do HEMOPA (238 aptos e 12 inaptos) e 31 do HRM e HG (todos aptos).

Pesquisa de plasmódios. Foram utilizadas as técnicas QBC Test $₫ 23$ e gota espessa.

Imunofluorescência para detecção de antígeno. Amostras de sangue total dos doadores foram centrifugadas, o sedimento foi lavado com PBS pH 7.2, ressuspendido em PBS contendo $4 \%$ de lactose e aplicado em lâminas de vidro. A reação foi realizada como descrito por Ávila1 com utilização de lgG humana anti-plasmódios (1/200). As lâminas foram observadas em microscópio de fluorescência com aumento de 250 vezes.

Obtenção de antígenos para reações sorológicas. As formas eritrocíticas de $P$. vivaxe $P$. falciparum foram obtidas de pacientes diagnosticados no Laboratório de Malária da Superintendência de Controle de Endemias (SUCEN). As amostras de P. vivax foram processadas em coluna de celulose CF11 para retirada de leucócitos 16 . Para concentração de formas maduras foi utilizado gradiente de Nycodenz ${ }^{\circledR}$ (Nycomed Pharma AS) a $58 \%$ em PBS na proporção de $1 / 519$. Formas eritrocíticas maduras de $P$. falciparum foram obtidas a partir de cultivo contínuo24. Para o ensaio imunoenzimático (ELISA), foi realizada concentração de esquizontes de $P$. falciparum com solução isotônica de gelatina a $3 \%$ (Plasmagel $\AA$ Laboratoire Roger Ballon)21. As lâminas para IFI foram preparadas imediatamente após a obtenção do material e estocadas a $-20^{\circ} \mathrm{C}$. As formas eritrocíticas de $P$. falciparum para o ELISA foram estocadas em nitrogênio líquido para posterior processamento com tampão TEN-Triton (Tris $10 \mathrm{mM}$ pH 8.0, EDTA $10 \mathrm{mM}, \mathrm{NaCl} 400 \mathrm{mM}$, Triton $\mathrm{X}-100$ a $2 \%$ ) para extração de antígenos solúveis.

Imunofluorescência indireta. Foi realizada com antígenos de $P$. vivax e $P$. falciparum, segundo técnica descrita por Ferreira e Sanchez ${ }^{13}$. Conjugado anti-IgG humana marcado com isotiocianato de fluoresceína (BIOLAB) foi titulado com soro de paciente malárico como controle positivo e soro de doador com sorologia negativa para malária, Chagas, sífilis, hepatites B e C, HIV, HTLV1, como controle negativo. O cut-off estabelecido para a reação foi de 1:40. As reações foram lidas em microscópio de fluorescência em aumento de 500 vezes. Cada soro foi testado em duplicata em experimentos independentes.

ELISA. O teste foi realizado para detecção de anticorpos IgG anti-P. falciparum conforme descrito por Voller25. A diluição ótima do antígeno e conjugado foi estabelecida por titulação em bloco utilizando soros controle positivo e negativo. O cut-off foi determinado a partir da média das densidades óticas de 20 soros negativos, acrescida de dois desvios-padrões. A leitura das placas foi realizada a $492 \mathrm{~nm}$ em leitor Auto Reader II (Ortho Diagnostic Systems). Cada soro foi testado em duplicata em experimentos independentes.

Método estatístico. Para o cálculo estatístico foi utilizado o teste de Fisher.

\section{RESULTADOS}

Os resultados obtidos na pesquisa de anticorpos antiplasmodiais por IFI e ELISA estão expressos na Tabela 1.

Área sem transmissão ativa. Na FPS/HSP foram colhidas 1200 amostras de sangue, sendo 1164 de doadores aptos e 36 de doadores inaptos. Destes últimos, 12 referiram episódio malárico há mais de 3 anos (de 4 a 40 anos) e 24 se deslocaram por áreas com risco de transmissão de malária. 
Tabela 1 - Positividade das amostras de sangue de doadores por IFI e ELISA, para pesquisa de anticorpos antiplasmodiais.

\begin{tabular}{|c|c|c|c|c|c|c|c|c|c|c|c|c|}
\hline \multirow{3}{*}{$\begin{array}{l}\text { Pesquisa de anticorpos } \\
\text { da classe IgG }\end{array}$} & \multicolumn{4}{|c|}{ IFI-IgG anti- $P$. vivax } & \multicolumn{4}{|c|}{ IFI-IgG anti- $P$. falciparum } & \multicolumn{4}{|c|}{ ELISA-IgG anti- $P$. falciparum } \\
\hline & \multicolumn{2}{|c|}{ positivo } & \multicolumn{2}{|c|}{ negativo } & \multicolumn{2}{|c|}{ positivo } & \multicolumn{2}{|c|}{ negativo } & \multicolumn{2}{|c|}{ positivo } & \multicolumn{2}{|c|}{ negativo } \\
\hline & $\mathrm{n}^{\circ} \mathrm{o}$ & $\%$ & $\mathrm{n}^{\circ}$ & $\%$ & $\mathrm{n}^{\circ}$ & $\%$ & $\mathrm{n}^{\circ}$ & $\%$ & $\mathrm{n}^{\circ}$ & $\%$ & $\mathrm{n}^{\circ}$ & $\%$ \\
\hline \multicolumn{13}{|l|}{$\overline{\text { São Paulo, SP }(n=1.200)}$} \\
\hline aptos $(n=1164)$ & 14 & 1,2 & 1150 & 98,8 & 10 & 0,9 & 1154 & 99,1 & 12 & 1,0 & 1152 & 99,0 \\
\hline inaptos $(n=36)$ & 6 & 16,7 & 30 & 83,3 & 2 & 5,6 & 34 & 94,4 & 1 & 2,7 & 35 & 97,3 \\
\hline \multicolumn{13}{|l|}{ Belém, PA $(n=250)$} \\
\hline aptos $(n=238)$ & 23 & 9,6 & 215 & 90,4 & 24 & 10,1 & 214 & 89,9 & 41 & 17,2 & 197 & 82,8 \\
\hline inaptos $(n=12)$ & 3 & 25,0 & 9 & 75,0 & 5 & 41,6 & 7 & 58,4 & 3 & 25,0 & 9 & 75,0 \\
\hline \multicolumn{13}{|l|}{ Matupá, MT (n = 31) } \\
\hline $\operatorname{aptos}(n=31)$ & 23 & 74,2 & 8 & 25,8 & 20 & 64,5 & 11 & 35,5 & 17 & 54,8 & 14 & 45,2 \\
\hline
\end{tabular}

A pesquisa de plasmódios e a imunofluorescência para detecção de antígenos foi negativa nos dois grupos (aptos e inaptos).

Os dados obtidos na IFI para pesquisa de anticorpos anti $P$. vivax e $P$. falciparum foram os seguintes: $1,98 \%$ das amostras do grupo dos doadores aptos foram positivas, sendo 13 positivas para $P$. vivax, 9 positivas para $P$. falciparum e 1 co-positiva para as duas espécies (Figura 1a); $22,3 \%$ das amostras do grupo dos doadores inaptos foram positivas, sendo 6 positivas para $P$. vivax e 2 positivas para $P$. falciparum (Figura 1b)

$\mathrm{Na}$ reação de ELISA-IgG anti- $P$. falciparum os resultados mostraram 12 amostras positivas que correspondem a $1 \%$ do total de aptos (Figura 1c). Para os doadores inaptos detectamos 1 amostra positiva (2,7\%) (Figura 1d).

Área de baixa transmissão. Das 250 amostras de sangue colhidas no HEMOPA, 238 foram de doadores aptos e 12 de inaptos. Destes últimos, 9 referiram deslocamentos por áreas com risco de transmissão de malária nos últimos 6 meses, 2 referiram malária anterior e um apresentou febre nos últimos 30 dias. Dos doadores aptos, $9(3,8 \%)$ tiveram um episódio de malária sendo 8 deles há mais de 3 anos.

A presença de plasmódios foi avaliada através de gota espessa, QBC Test $₫$ e imunofluorescência para pesquisa de antígenos, sendo todas as amostras negativas.

Os dados obtidos na IFI para pesquisa de anticorpos anti- $P$. vivax e anti- $P$. falciparum foram os seguintes: $17,2 \%$ das amostras do grupo dos doadores aptos foram positivas, sendo 17 positivas para $P$. vivax, 18 positivas para $P$.
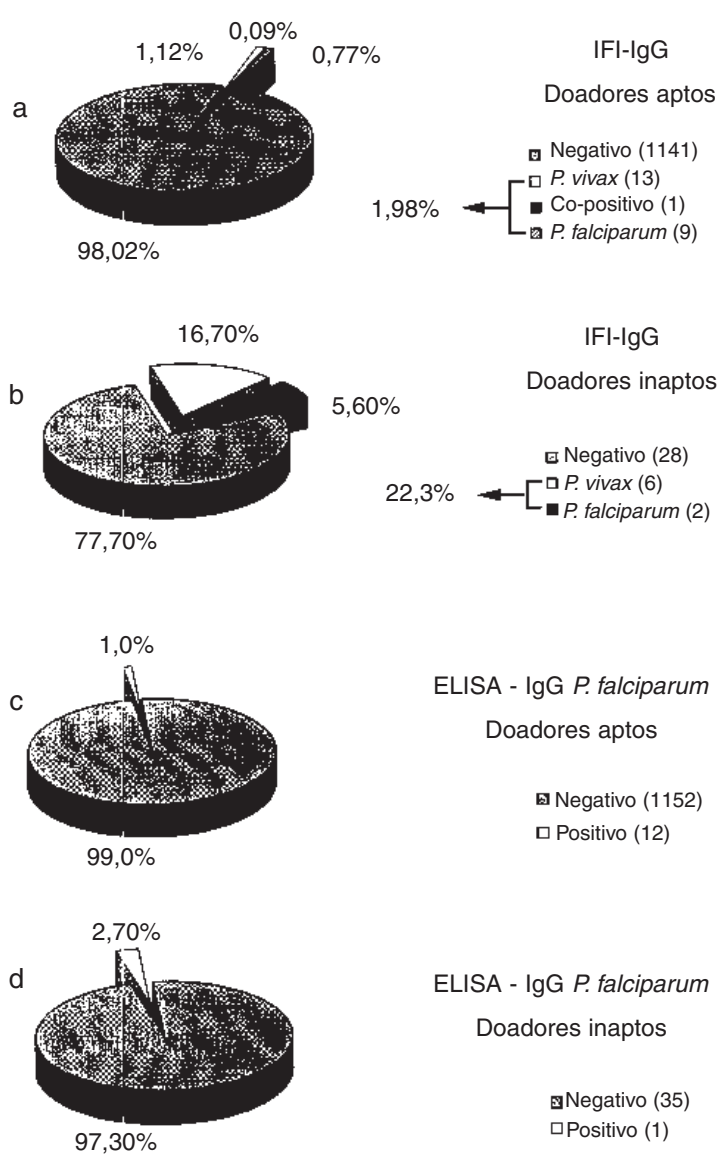

ELISA - IgG P. falciparum

Doadores aptos

Negativo (1152)

$\square$ Positivo (12)

ELISA - IgG P. falciparum

Doadores inaptos

QNegativo (35) $\square$ Positivo (1)

Figura 1 - Controle da malária transfusional em região endêmica e não endêmica do Brasil. 
falciparum e 6 co-positivas para as duas espécies (Figura 2a); $58,3 \%$ das amostras do grupo dos doadores inaptos foram positivas, sendo 2 para $P$. vivax, 4 para $P$. falciparum e 1 co-positiva para as duas espécies (Figura 2b).

No teste ELISA anti-P. falciparum os resultados mostraram 41 amostras positivas que correspondem a $17,2 \%$ do total de aptos (Figura 2c). Para os doadores inaptos detectamos 3 amostras positivas (25\%) (Figura 2d).

Área de alta transmissão. Das 31 amostras de sangue analisadas, 27 foram colhidas no HRM e 4 no HG. Dos 31 doadores, 30 referiram episódio malárico anterior, sendo: de 1 a 2 episódios de malária, 8 doadores; de 3 a 10 episódios de malária, 8 doadores e com mais de
10 episódios de malária, 14 doadores. Com relação ao tempo decorrido desde o último episódio de malária, temos: menos de 1 ano, 3 doadores; de 1 a 3 anos, 14 doadores; mais de 3 anos, 13 doadores.

$\mathrm{Na}$ pesquisa de plasmódios e na imunofluorescência para detecção de antígenos, todas as amostras foram negativas.

Através da IFI para pesquisa de anticorpos, $80,6 \%$ das amostras foram positivas, sendo 5 para $P$. vivax, 2 para $P$. falciparum e 18 copositivas para as duas espécies (Figura $3 a$ ).

Através do teste ELISA-IgG anti-P. falciparum, 14 amostras foram negativas $(45,2 \%)$ e 17 foram positivas $(54,8 \%)$ (Figura $3 b)$. a

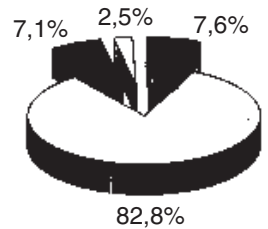

b

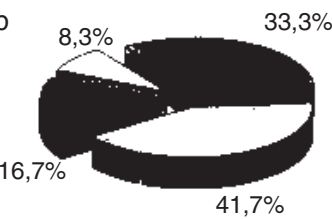

c
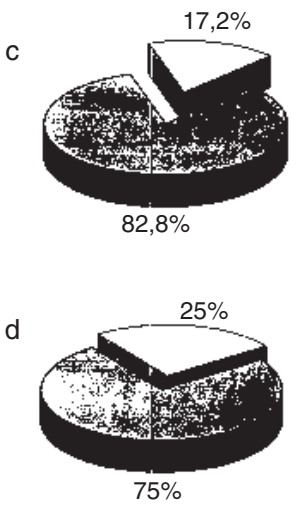

IFI-IgG

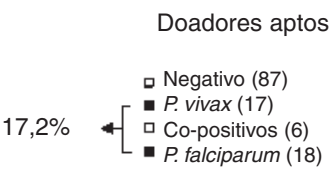

IFI-IgG

Doadores inaptos

- Negativo (5)

- P. vivax (2)

$58,3 \%+$ Co-positivos (1)

- P. falciparum (4)

ELISA - IgG P. falciparum

Doadores aptos

口 Negativo (197)

$\square$ Positivo (41)

ELISA - IgG P. falciparum

Doadores inaptos

- Negativo (9)

口 Positivo (3) b

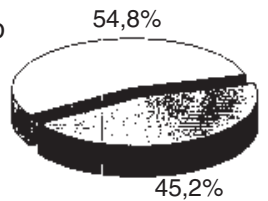

Figura 3 - Controle da malária transfusional em região

ELISA - IgG P. falciparum

口 Negativo (14)

п Positivo (17)

Figura 2 - Controle da malária transfusional em região

a

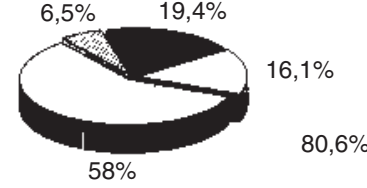

Privo (6)

- P. vivax (5)

- Co-positivos (18) 


\section{DISCUSSÃO}

Apesar da alta prevalência de $P$. vivax no Brasil $(64,6 \%$ dos casos de 1994) e da maior freqüência de malária transfusional ocasionada por esta espécie7, diversos estudos realizados entre doadores de sangue utilizaram nas provas sorológicas somente antígeno de $P$. falciparum, que pode ser obtido a partir de cultivo contínuo. Embora ocorra reação cruzada entre as espécies com a utilização de antígenos heterólogos, pode haver falha na detecção de anticorpos anti$P$. vivax 9 . Há necessidade de desenvolvimento de testes laboratoriais rápidos, capazes de detectar antígenos para as diferentes espécies de Plasmodium, de fácil execução e com custo acessível a países em desenvolvimento, de modo a possibilitar seu emprego em áreas com infraestrutura deficiente. Também que possuam alta sensibilidade, pois portadores assintomáticos, com baixa parasitemia no momento da doação, são os que constituem problema para a triagem de doadores em bancos de sangue 26 .

Considera-se importante a triagem de sangue e hemocomponentes a serem administrados principalmente em imunodeprimidos, pois estudos anteriores demonstraram que, em pacientes esplenectomizados tratados com corticosteróides, imunossupressores ou radioterapia para tumores malignos e discrasias sangüíneas, a eventual transmissão de malária pode acarretar sintomas severos com envolvimento cerebral precoce 17 .

A escolha entre os testes, gota espessa ou QBC Test ${ }^{\circledR}$, deverá ser decorrente da disponibilidade de recursos e infra-estrutura, mas sempre levando em consideração que: a gota espessa, apesar de sua eficiência, praticidade e baixo custo, detecta 10 parasitas por $\mu \mathrm{l}$ de sangue 8 e o QBC Test $\circledast$ permite a detecção de $10 \%$ mais infecções que a microscopia convencional23.

Nossos resultados mostram grande variação nos índices de positividade para presença de anticorpos antiplasmodiais por IFI-lgG anti $P$. vivax e anti $P$. falciparum e ELISA-IgG anti- $P$. falciparum entre doadores aptos em diferentes regiões do Brasil. Em Matupá e Peixoto de Azevedo, municípios situados na região Centro-Oeste ao norte do Estado de Mato Grosso, a positividade foi de 80,6\%; em BelémPA, município situado na região Norte foi de $17,2 \%$ e em São Paulo-SP, município localizado na região Sudeste foi de 1,98\%. Embora saibamos que a presença de anticorpos é um parâmetro que não confirma infecção malárica ativa, ficam caracterizadas distintas populações de doadores. Portanto, os critérios estabelecidos para triagem epidemiológica e laboratorial também precisam ser diferenciados.

A validade da triagem clínico epidemiológica foi confirmada pela diferença observada por IFI - IgG anti $P$. vivax e anti $P$. falciparum entre os grupos de doadores aptos e inaptos nos hemocentros de São Paulo e Belém, pelo teste de Fisher $(p<0,01)$. Entre os doadores de São Paulo a positividade foi de $1,98 \%$ no grupo dos aptos e de $22,3 \%$ no grupo dos inaptos e em Belém foi de $17,2 \%$ e $58,3 \%$, respectivamente. Nos municípios de Matupá e Peixoto de Azevedo, como o único critério utilizado para exclusão foi a presença de febre nos últimos 30 dias, não houve doadores inaptos. Nota-se a extrema precariedade da triagem, pois nenhum teste para pesquisa de plasmódios é realizado entre doadores da região. Além disso, é difícil a caracterização de malária transfusional, pois como há transmissão urbana, os eventuais casos de malária póstransfusional, em geral são considerados decorrentes de transmissão vetorial. Esta situação, provavelmente, não é peculiar apenas nesses dois municípios onde o estudo foi realizado, mas deve refletir as condições de grande número de municípios da Região Amazônica, onde a malária é endêmica.

A estrutura deficitária dos serviços de hemoterapia oferecida à população de região endêmica precisa ser corrigida pela expansão das hemorredes ou pelo suprimento regular de unidades de sangue e hemocomponentes devidamente triados, não apenas para malária, como para outras patologias passíveis de transmissão por transfusão sangüínea. Deve-se também considerar que a malária grave ocasionada por P. falciparum, com altas parasitemias e conseqüente destruição de grande número de hemácias, é causa freqüente de prescrição de transfusão sangüínea ou de tratamento por exsangüíneo transfusão.

$\mathrm{Em}$ áreas sem transmissão ativa ou com focos esporádicos de malária, a triagem clínico-epidemiológica rigorosa, abordando aspectos tais como febre recente, deslocamento por áreas com transmissão, malária anterior, uso de profiláticos, uso de drogas injetáveis e transfusão sangüínea, constitui o mais eficiente mecanismo de exclusão de doadores. Estudo retrospectivo realizado nos Estados Unidos, 
constatou que em $50 \%$ dos casos de malária transfusional, onde o doador do sangue infectado foi identificado, poderiam ter sido evitados através da triagem clinico-epidemiológica22. Nessas regiões, no caso de doações específicas para pacientes imunossuprimidos, quando for necessário trabalhar com amostras de indivíduos provenientes de áreas com transmissão ativa, recomenda-se a utilização de testes para detecção de parasitas (QBC Test® e/ou gota espessa) ou detecção de antígenos parasitários por imunofluorescência ou ELISA2 10.

Em áreas com baixa transmissão, a elevada positividade (17,2\% no HEMOPA) para anticorpos IgG por IFI utilizando-se antígenos de $P$. vivax e $P$. falciparum sugere a necessidade de introdução de testes para pesquisa de plasmódios, além do seguimento das normas estabelecidas pelo Ministério da Saúde para triagem clínicoepidemiológica, embora nesse estudo não tenham sido detectadas amostras positivas pelo QBC Test ${ }^{\circ}$, gota espessa ou pesquisa de antígenos por imunofluorescência.

Em áreas com alta transmissão, onde a triagem clínico-epidemiológica não pode ser rigorosa, pois isto acarretaria a exclusão de grande número de candidatos a doadores de sangue, torna-se fundamental a inclusão de testes laboratoriais para pesquisa de plasmódios

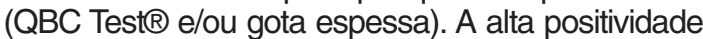
dos testes sorológicos para detecção de anticorpos, inviabiliza sua utilização como instrumento de triagem nestas áreas, visto que o índice de exclusão pode exceder $80 \%$, como demonstrado pelos resultados obtidos neste estudo.

\section{REFERÊNCIAS BIBLIOGRÁFICAS}

1. Ávila SLM. Avaliação do desempenho de métodos de laboratório no diagnóstico da malária humana: sugestões para normatização. Tese de doutorado, Instituto de Ciências Biomédicas, Universidade de São Paulo, 1994.

2. Ávila SLM, Leandro MC, Arruk VG, Carvalho NB, Oliveira MS, Sanchez MCA, Boulos M, Ferreira AW. Evaluation of different methods for Plasmodia detection in well defined population groups in an endemic area of Brazil. Revista do Instituto de Medicina Tropical de São Paulo 36:157-162, 1994.

3. Brasil. Ministério da Saúde, Fundação Nacional de Saúde, Gerência Técnica de Malária,Brasília, Dados epidemiológicos de malária por município, 1993.
4. Brasil. Ministério da Saúde, Fundação Nacional de Saúde, CENEPI, Brasília, Informe Epidemiológico do SUS, 1995.

5. Brasil. Superintendência de Controle de Endemias. Secretaria de Estado da Saúde do Estado de São Paulo. Relatório de Avaliação do Programa de Controle de Malária, 1995.

6. Bruce-Chwatt LJ. Transfusion malaria. Bulletin of the World Health Organization 50:337-346, 1974.

7. Bruce-Chwatt LJ. Transfusion malaria revisited. Tropical Diseases Bulletin 34:827-840, 1982.

8. Bruce-Chwatt LJ. Essential Malariology, $2^{\text {nd }}$ edition. London, William Heinemann Medical Books, 1985.

9. Carvalho ME, Ferreira MU, Souza MRD, Ninomia RT, Matos GF, Camargo LMA, Ferreira CS. Malaria soroepidemiology: comparison between indirect fluorescent antibody test and enzyme immunoassay using bloodspot eluates. Memórias do Instituto Oswaldo Cruz 87:205-208, 1992.

10. Choudhury N, Jolly JG, Ganguly NK, Mahajan RC, Dubey ML. Plasmodial antigen detection by monoclonal antibody as a screening procedure for blood donors in transfusion medicine. Journal of the Indian Medical Association 89:334-336, 1991.

11. Cliquet MG. A triagem clínica de doadores de sangue como método de prevenção da transmissão de doenças através das transfusões. Hematologia e Hemoterapia 1:10-13, 1996.

12. Dover AS, Guinee VF. Malaria transmission by leukocyte component therapy. Journal of the American Medical Association 217:1701-1702, 1971.

13. Ferreira AW, Sanchez MCA. Malária humana. Padronização e optimização de testes sorológicos para diagnóstico individual e inquéritos soroepidemiológicos. Revista do Instituto de Medicina Tropical de São Paulo 30:137-146, 1988.

14. Garfield MD, Ershler WB, Dennis GM. Malaria transmission by platelet concentrate transfusion. Journal of the American Medical Association 240:2285-2286, 1978.

15. Guerrero IC, Weniger BC, Schultz MG. Transfusion malaria in United States, 1972-1981. Annals of Internal Medicine 99:221-226, 1983.

16. Homewood CA, Neame KD. A comparison of methods used for removal of white cells from malaria infected blood. Annals of Tropical Medicine and Parasitology 70:249-251, 1976.

17. Joishy SK, Lopez CG. Transfusion-induced malaria in a splenectomized B-thalassemia major patient and review of blood donor screening methods. American Journal of Hematology 8:221-229, 1980. 
18. Lozner EL, Newhouser LR. Studies on the transmissibility of malaria by plasma transfusion. In: American Journal of Medical Science 206:141-146, 1943, Apud Sazama, p.786. 1991.

19. Mons B, Croon JJAB, Van Der Star W, Van Der Kaay HJ. Erythrocytic schizogony and invasion of Plasmodium vivax in vitro. International Journal for Parasitology 18:307-311,1988.

20. Ramos AMSV, Sáez-Alquézar A, Di Santi SM, Branquinho MS, Kirchgatter K, Ferrari L, Cliquet MG, Moraes ACP, Cordeiro IAC, Bracco L, Salles NA, Dorliac-Llacer PE, Chamone DAF. Métodos de detecção de plasmódios e anticorpos antimaláricos em doadores da Fundação Pró-Sangue Hemocentro de São Paulo. In: Resumos do XXI Congresso Brasileiro de Hematologia e Hemoterapia, São Paulo p.134, 1994.

21. Reese RT, Langreth SG, Trager W. Isolation of stages of human parasite Plasmodium falciparum from culture and from animal blood. Bulletin of the World Health Organization 57 (suppl):53-61, 1979.
22. Sazama K. Prevention of transfusion - transmitted malaria: Is it time to revisit the standards? Transfusion 31:786-788, 1991.

23. Spielman A, Perrone JB. Malaria diagnosis by direct observation of centrifuged samples of blood. American Journal of Tropical Medicine and Hygiene 39:337-342, 1988.

24. Trager W, Jensen JB. Human malaria parasites in continuous culture. Science 193:673-675, 1976.

25. Voller A, Bidwell D, Huldt G, Engvall E. A microplate method of enzyme-linked immunosorbent assay and its application to malaria. Bulletin of the World Health Organization 51:209-211, 1974.

26. Wells L, Ala FA. Malaria and blood transfusion. Lancet 1:1317-1319, 1985.

27. Woolsey G. Transfusion for pernicious anaemia: two cases. In: Annals of Surgery 53:132-135, 1911. Apud Sazama, p.786, 1991. 